\title{
Cuadro de mando integral (CMI) de los Centros de Salud Familiar del Departamento de Salud Municipal de la comuna de Puerto Montt, Chile
}

NANCY ALARCÓN-HENRÍQUEZa , JOSÉ VERA-GARNICA

RESUMEN El uso del cuadro de mando integral (CMI) ha aumentado de manera creciente en el sector salud en Chile. A partir del 2006, comenzaron a emplearlo diversos Departamentos de Salud Municipales y Centros de Salud Familiar (Cesfam) dependientes de esos departamentos.

El CMI es un modelo de gestión que traduce la estrategia en objetivos relacionados, medidos a través de indicadores, y ligados a unos planes de acción que permiten alinear el comportamiento de los miembros de la organización. La utilidad del CMI no depende del tipo de empresa sino de los problemas que enfrenta, y de hecho se ha implementado en grandes y pequeñas empresas, y en organizaciones con y sin ánimo de lucro, tal como se demuestra en este caso.

Se presenta en este estudio el desarrollo de la planificación estratégica de los Cesfam de la comuna de Puerto Montt, Chile, empleando el cuadro de mando integral (CMI) o Balanced Scorecard (BSC).

La implementación del modelo implicó el trabajo y compromiso de los equipos gestores de cada uno de los Cesfam en el proceso de planificación estratégica y la definición de la totalidad de los componentes del cuadro de mando.

PALABRAS CLAVE estrategia, gestión, planificación, salud pública.

\section{HISTORIA DEL ARTÍCULO}

¿CÓMO CITAR?:

Alarcón-Henríquez, N. \& VeraGarnica, J. (2015). Cuadro de mando integral (CMI) de los Centros de Salud Familiar del Departamento de Salud Municipal de la comuna de Puerto Montt, Chile. Perspectiva Empresarial, 2(2), 97-108. http:// dx.doi.org/10.16967\%2Frpe.v2n2a7

RECIBIDO: 4 de junio de 2015 APROBADO: 21 de septiembre de 2015

CORRESPONDENCIA:

José Vera Garnica, Chinquihue km 6, Universidad de Los Lagos, Puerto Montt, Chile.

a Doctoranda en Gestión de Negocios Internacionales, Universidad de Lleida, España. Académica de jornada completa, Universidad de Los Lagos, Chile. Correo electrónico: n.alarcon@ulagos.cl

b Doctorando en Estudios Empresariales, Universidad de Barcelona, España. Académico de jornada completa, Universidad de Los Lagos, Chile. Correo electrónico: jvera@ulagos.d 


\section{Balanced Scorecard (BSC) of family health centers attached to the municipal health department in the commune of Puerto Montt, Chile}

ABSTRACT The use of Balance Scorecards (BSC) has gradually increased in the Chilean health sector. Since 2006, it has been used by various Municipal Health Departments and Family Health Centers (Cesfam) attached to such departments.

The BSC is a management model that translates strategy into related objectives that are measured by indicators and linked to certain action plans that allow to adjust the behavior of the members of an organization. The usefulness of BSC does not depend on the type of company but on the problems it faces. In fact, it has been implemented in both small and large companies and for-profit and non-profit organizations, as shown in this case.

This study discusses the development of Cesfam strategic planning in the commune of Puerto Montt, Chile, using the Balance Scorecard (BSC).

The implementation of this model involved the work and commitment of each Cesfam's managing team in the strategic planning process and the determination of all components in the Balance Scorecard.

KEYWORDS strategy, management, planning, public health.

\section{Balanced Scorecard dos centros de saúde familiar do departamento de saúde municipal da comunidade de Puerto Montt, Chile}

RESUMO O uso do Balanced Scorecard (BSC) vem aumentando de maneira crescente no setor de saúde no Chile. A partir de 2006, diversos Departamentos de Saúde Municipais e Centros de Saúde Familiar (Cesfam) dependentes desses departamentos começaram a utilizá-lo.

O BSC é um modelo de gestão que traduz a estratégia em objetivos relacionados, medidos por meio de indicadores e ligados a planos de ação que permitem alinhar o comportamento dos membros da organização. A utilidade do BSC não depende do tipo de empresa, mas sim dos problemas que enfrenta e, de fato, vem sendo implantado em grandes e pequenas empresas, além de organizações com ou sem fins lucrativos, tal como se demonstra neste caso.

Apresenta-se, neste estudo, o desenvolvimento do planejamento estratégico dos Cesfam da comunidade de Puerto Montt (Chile) utilizando o BSC.

A implantação do modelo implicou o trabalho e o compromisso das equipes gestoras de cada um dos Cesfam no processo de planejamento estratégico e na definição da totalidade dos componentes do BSC.

PALAVRAS CHAVE estratégia, gestão, planejamento, saúde pública. 


\section{Introducción}

El modelo de atención primaria en salud es una propuesta de atención integral adaptada a las nuevas demandas de salud de la comunidad, que ha aprobado el Ministerio de Salud en Chile (Superintendencia de salud, 2009).

Hoy en día, la experiencia médica, por sí sola, no alcanza a dar cuenta de la complejidad de los problemas de salud que afectan a la sociedad, caracterizados por la gran cantidad de factores de la vida moderna que afectan la salud humana.

Por tal razón, se hace necesario complementar una visión integral de las distintas disciplinas que interactúan en el bienestar de las personas y promover el cuidado de la salud, mejorar la calidad de vida, prevenir factores de riesgo y -quizás lo más importante-favorecer el cambio de rol, de "paciente" a "sujeto participante" en el cuidado y prevención de su salud.

El nivel primario de atención en salud se caracteriza por ser la atención que ofrecen los consultorios y postas rurales a través de todo el país. Se considera la puerta de entrada al sistema público de salud, lo que representa el primer contacto de los individuos, la familia y la comunidad con el sistema de salud, brindando una atención ambulatoria a través de:

- Centros de Salud (CES)

- Centros de Salud Familiar (Cesfam)

- Centros Comunitarios de Salud Familiar (Cecosf)

- Postas Salud Rurales (PSR)

- SAPU (Servicio de Atención Primaria de Urgencia)

Estos Centros de Salud son administrados en su totalidad por las municipalidades, puesto que son servicios traspasados, según lo que establece la Ley No.18.695 Orgánica Constitucional de Municipalidades, de acuerdo con lo estipulado en el DFL No. 1-3063 de 1980.

El objetivo de la atención primaria es otorgar una atención equitativa y de calidad, centrada en las personas y sus familias, enfocada en lo preventivo y promocional, es decir, anticipándose a la enfermedad, acorde con el modelo de salud integral con enfoque familiar y comunitario (Ministerio de Salud, Subsecretaría de Redes Asistenciales, 2008).

Los Centros de Salud Familiar proporcionan cuidados básicos en salud, con acciones de promoción, prevención, curación, tratamiento, cuidados domiciliarios y rehabilitación de la salud, y atienden en forma ambulatoria. Un Cesfam trabaja según el modelo de salud integral con enfoque familiar y comunitario; da mayor énfasis a la prevención y promoción de salud; se centra en las familias y la comunidad; da importancia a la participación comunitaria; trabaja con un equipo de salud de cabecera que atiende a toda la familia en salud y enfermedad durante todo el ciclo vital, y con estas acciones pretende mejorar la calidad de vida de las personas (Ministerio de Salud, 2012).

\section{Planificación estratégica en la administración pública chilena}

La planificación estratégica, según Hax \& Majluf (1996), tiene que ver con el efecto futuro de las decisiones tomadas hoy. Parte esencial es la identificación de oportunidades y amenazas en el medio ambiente en que se desenvuelve la institución, y su contraste con las fortalezas y debilidades de la organización. Entre otros aspectos consiste en decidir anticipadamente qué se hará y de qué manera; esto incluye diferentes aspectos, que van desde la determinación de objetivos de carácter prioritario hasta políticas de desarrollo, incorporando la fijación de metas, programas y acciones para lograrlo. Para Armijo (2009), una característica principal del proceso estratégico es el establecimiento de los cursos de acción (estrategias) ${ }^{1}$ para alcanzar dichos objetivos.

La planificación estratégica es una herramienta clave para el apoyo a la gestión pública, cuyo proceso se inició a mediados de los años 1980 en los países de la OCDE, en el marco de las iniciativas de la nueva gerencia pública o New Public Management. Su aplicación en el ámbito público se concibe como una herramienta imprescindible para la identificación de prioridades y asignación de recursos en un contexto de cambios y altas

1 El término "estrategia" se deriva del griego "strategos", que significa, literalmente, "general del ejército". Cada una de las diez tribus de la antigua Grecia elegía cada año un strategos para dirigir su regimiento. En la batalla de Maratón (490 a. C.), los strategos asesoraron al gobernante político como un consejo. Dieron asesoría "estratégica" sobre la gestión de las batallas para ganar las guerras, en lugar de asesorar sobre "tácticas" de manejo de las tropas para ganar las batallas.

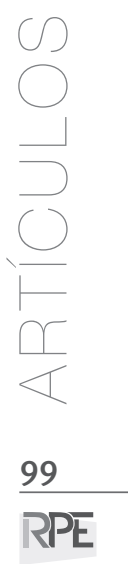


exigencias por avanzar hacia una gestión comprometida con los resultados.

Las metodologías para desarrollar procesos de planificación estratégica son variadas, y se encuentran en la literatura diversos enfoques. En su aplicación a las organizaciones públicas no existe un modelo a seguir, y es posible encontrar metodologías que tienen mayor o menor complejidad (Salinas M. et al., 2012). Entre los modelos aplicados en la administración pública chilena se destaca el Balanced Scorecard (BSC), (Leiva H. \& Flamm M., 2007).

En la simplificación del proceso estratégico para la administración pública chilena, la Dirección de Presupuesto (Dirpres) proporciona definiciones estratégicas, que corresponden a una herramienta que entrega información sobre los ejes orientadores del quehacer de una organización; se obtienen a partir de un proceso de planificación estratégica o de un proceso más simple de diagnóstico, análisis, reflexión y toma de decisiones colectivas en torno al quehacer actual de la institución. La información corresponde a la misión, objetivos estratégicos, productos estratégicos (bienes o servicios) y usuarios o beneficiarios. Su incorporación tiene por objeto apoyar los programas de mejoramiento de la gestión (PMG) de las instituciones públicas chilenas, proceso que tiene su origen en la Ley No. 19.553 de 1998.

\section{Objetivos}

Describir la realización del Plan Estratégico de los Cesfam del Departamento de Salud Municipal de Puerto Montt, Chile, empleando la metodología de cuadro de mando integral o Balanced Scorecard.

\section{Material y métodos}

La herramienta empleada para el desarrollo de la planificación estratégica de los Centros de Salud Familiar (Cesfam) es el cuadro de mando integral (CMI) o Balanced Scorecard.

El cuadro de mando integral es un modelo estratégico-operacional que permite desarrollar, implementar y controlar la aplicación de una estrategia en una organización, dado que traduce la estrategia en objetivos relacionados, medidos a través de indicadores, y ligados a unos planes de

FIGURA 1. Cuadro de mando integral (CMI), las cuatro perspectivas

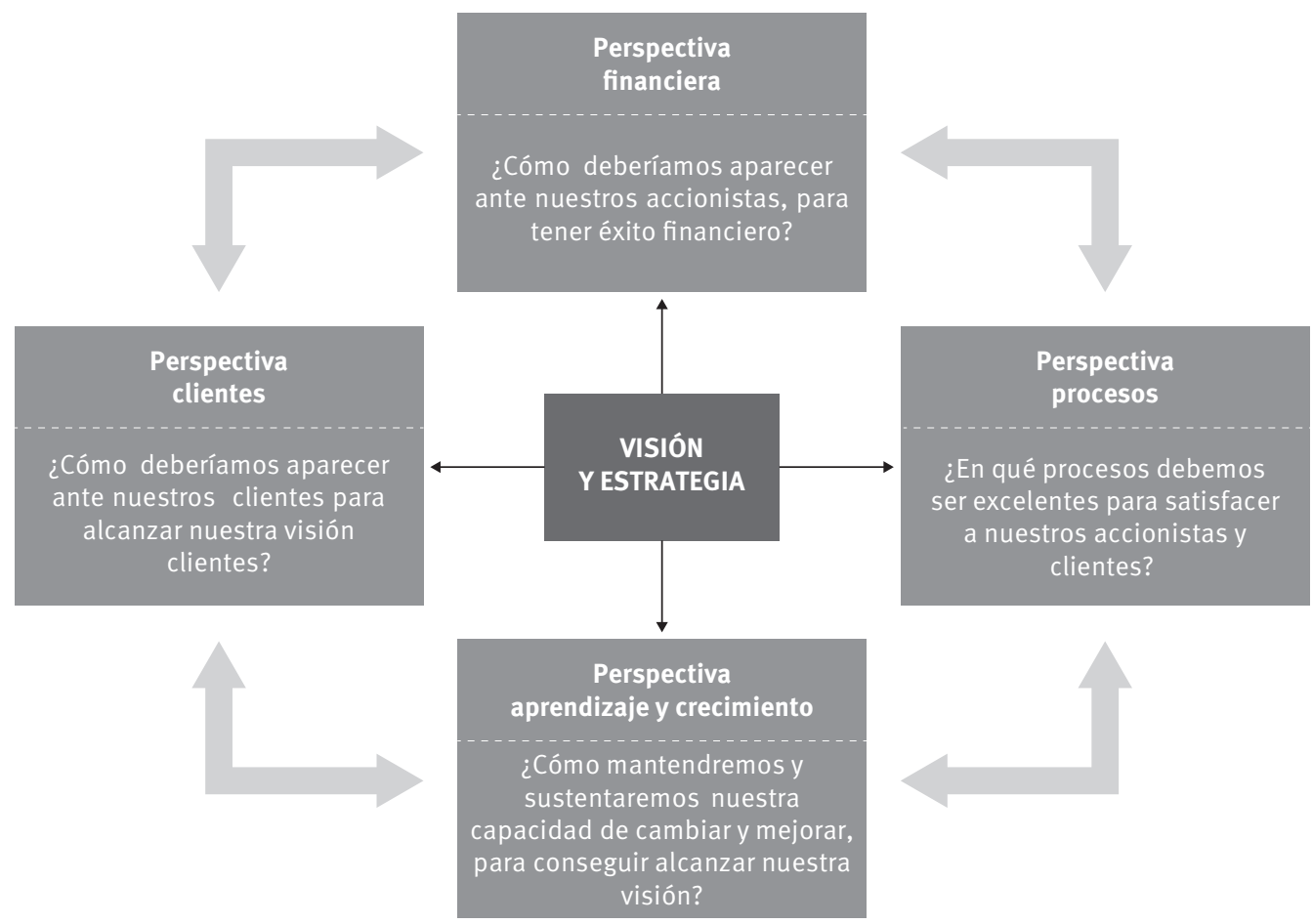


acción que permiten alinear el comportamiento de los miembros de la organización.

Conceptualmente, el Balanced Scorecard es una herramienta de gestión estratégica, que ofrece una visión integrada y balanceada de la organización, y permite desarrollar la estrategia en forma clara; para ello obliga a definir estrategias corporativas, revisar los procesos clínicos y administrativos, y definir los objetivos estratégicos que servirán de guía para el comportamiento y el desempeño de los equipos directivos en salud (Benavente, 2012).

El modelo está compuesto por:

- Las declaraciones estratégicas corporativas que marcan el rumbo de la organización.

- Un conjunto de objetivos estratégicos dispuestos en cuatro perspectivas.

- Un conjunto de indicadores asociados a los objetivos.

- Una direccionalidad de referencia hacia donde se pretende llevar o movilizar cada indicador.

- Un conjunto de acciones por realizar para alcanzar el valor esperado de cada indicador.

Metodológicamente, la Planificación Estratégica 2014-2016 fue desarrollada en tres etapas:

1. Análisis estratégico: consistió en trabajo de campo para recopilar información y su correspondiente análisis y sistematización.

2. Desarrollo de planificación: correspondió al desarrollo de los talleres de planificación, con la participación de los equipos gestores, en los cuales se definieron los objetivos estratégicos para cada Cesfam y se construyeron las respectivas matrices de planificación que forman parte de los planes de acción 2014-2016.

3. Diseño de un sistema de seguimiento compuesto por tres elementos: una estructura funcional de seguimiento y control, un conjunto de herramientas de control de gestión y un sistema de información diseñado especialmente para ese fin.

\section{Análisis estratégico}

El análisis consistió en:

- Revisión documental del Pladeco institucional, Revisión del Plan Comunal de Salud 20132016.
- Revisión del Modelo de Atención con enfoque familiar en la atención primaria, con revisión de la pauta de certificación, específicamente en los cinco Centros de Salud Familiar, dependientes de la Municipalidad de Puerto Montt.

- Centro de Salud Familiar Angelmó

- Centro de Salud Familiar Antonio Varas

- Centro de Salud Familiar Alerce

- Centro de Salud Familiar Carmela Carvajal

- Centro de Salud Familiar Padre Hurtado

- Levantamiento diagnóstico de la infraestructura de cada uno de los Cesfam, mediante observación y entrevistas con usuarios.

- Levantamiento diagnóstico de la gestión interna y operatoria de cada Centro de Salud Familiar, en concordancia con el modelo de salud familiar, mediante observación y entrevistas con usuarios y personal de los Cesfam.

\section{Desarrollo de planificación}

Corresponde al desarrollo de talleres de trabajo, sistematización de la información y priorización de las propuestas de los informantes clave, con el objeto de elaborar los siguientes elementos:

Definición de misión, visión, valores, mapa estratégico y cuadro de mando

Para la definición de la misión, visión y valores se efectuaron dos talleres, en cada uno de los Centros de Salud Familiar. El primero de ellos convocó a los equipos gestores y a un grupo de funcionarios de cada Cesfam ${ }^{2}$. El taller comenzó con una exposición respecto de los aspectos teóricos básicos que contempla la elaboración de la planificación estratégica por parte de una organización. Se dio un especial énfasis a la definición de la misión, visión y valores como elementos de base de dicho proceso y a la metodología del Balanced Scorecard. La exposición se apoyó con elementos audiovisuales. La segunda parte de este taller consistió en distribuir a los participantes en grupos de cinco personas, para que en conjunto

2 Los funcionarios participantes en este taller fueron elegidos por cada Cesfam. 
redactaran lo que ellos pensaban era la misión, visión y valores de su Cesfam.

El segundo taller convocó a los equipos gestores de cada Cesfam, quienes acordaron efectuar una única declaración de misión, visión y valores para el conjunto de los Centros de Salud.

Para la determinación de los objetivos estratégicos y la construcción de los correspondientes mapas estratégicos se llevaron a cabo dos talleres con los equipos gestores de cada uno de los Cesfam.

Una vez definidos los mapas estratégicos, se trabajó, también en la modalidad de taller, en la construcción de las matrices de planificación cuyo formato fue definido para la presente consultoría. Tales matrices constituyen el cuadro de mando, con objetivos, metas, responsables e indicadores, además de criterios de alerta.

Se efectuaron tres talleres de trabajo con los equipos gestores. Paralelamente, cada equipo gestor destinó tiempo adicional para completar y revisar sus matrices.

Para la difusión de la planificación estratégica, se realizaron tres jornadas de socialización de acuerdo con el avance de la consultoría, apoyadas con material audiovisual e impreso.

\section{Sistema de seguimiento}

Resultaba imprescindible para el seguimiento y control del Plan contar con un sistema especialmente diseñado para tal efecto; por tal razón se desarrolló para cada Cesfam un software computacional basado en planillas Excel y uso de macros que permite que el control y seguimiento de la planificación y el cálculo de los indicadores respectivos.

\section{Resultados y discusión}

A continuación se presenta el resultado de la Planificación Estratégica de los Cesfam del Departamento de Salud Municipal de Puerto Montt, Chile, empleando la metodología de cuadro de mando integral o Balanced Scorecard.

Los directivos de cada Centro de Salud puedan visualizar la Planificación Estratégica 2014-2016 por medio de un despliegue de menús en intranet para facilitar su conocimiento y consulta.

A continuación se presentan las imágenes del software, con el Cesfam de Angelmó.

\section{FIGURA 2. Portada}

\section{BALANCED SCORECARD (BSC)}

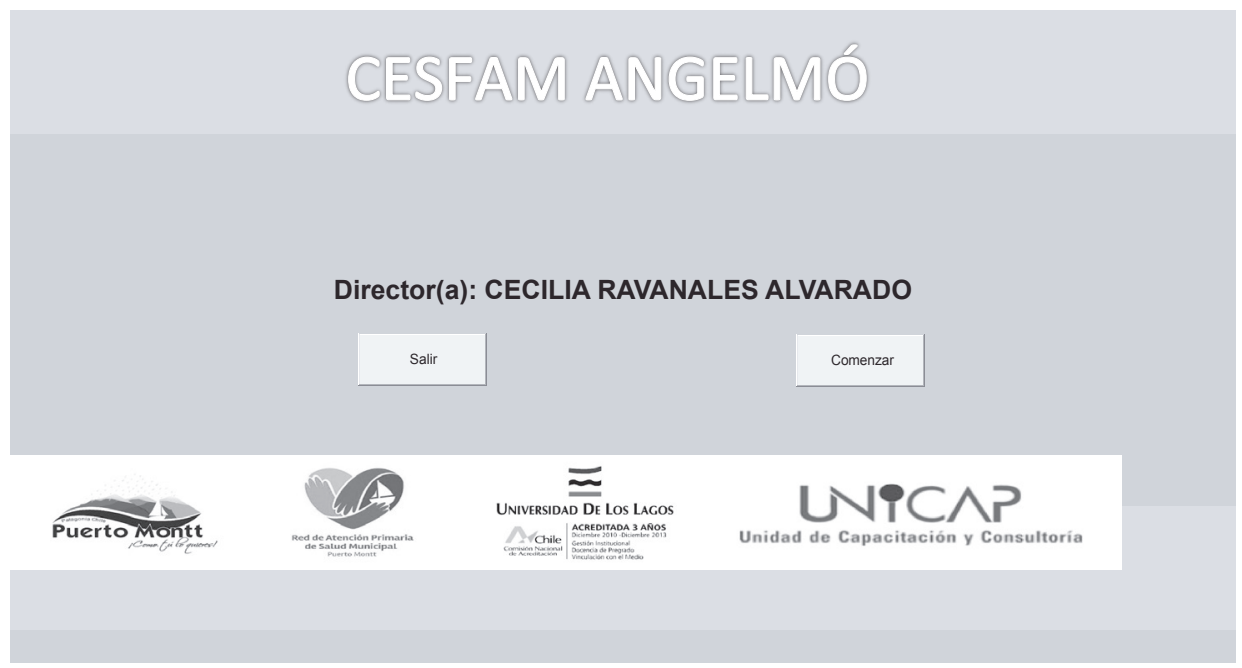


FIGURA 3. Menú principal

\begin{tabular}{|c|c|c|}
\hline \multicolumn{1}{|c|}{} & \multicolumn{1}{|c|}{ Misión y Visión } & Ver detalles \\
\hline & Mapa estratégico & Ver detalles \\
\hline P1 & Perspectivas & Ver detalles \\
\hline P2 & Perspectiva clientes/usuarios & Ver detalles \\
\hline P3 & Perspectiva financiera & Ver detalles \\
\hline P4 & Perspectiva procesos & Ver detalles \\
\hline
\end{tabular}

FIGURA 4. Misión y visión

号

SOMOS CENTROS DE ATENCIÓN PRIMARIA DE SALUD, CON ENFOQUE EN SALUD FAMILIAR Y COMUNITARIO, QUE ACOGEN, RESUELVEN YIO DERIVAN LAS NECESIDADES DE LOS USUARIOS DEL SISTEMA PÚBLICO DE PUERTO MONTT A LO LARGO DE SU CICLO VITAL, CON ÉNFASIS EN LA PROMOCIÓN, PREVENCIÓN Y EL ROL ACTIVO EN EL AUTOCUIDADO, CONSTITUIMOS EQUIPOS INTERDISCIPLINARIOS QUE BUSCAN CONTRIBUIR A ELEVAR LA CALIDAD DE VIDA DE LA POBLACIÓN. NUESTRA GESTIÓN ESTÁ BASABA EN EL COMPROMISO, CALIDAD, VOCACIÓN DE SERVICIO Y TOLERANCIA.

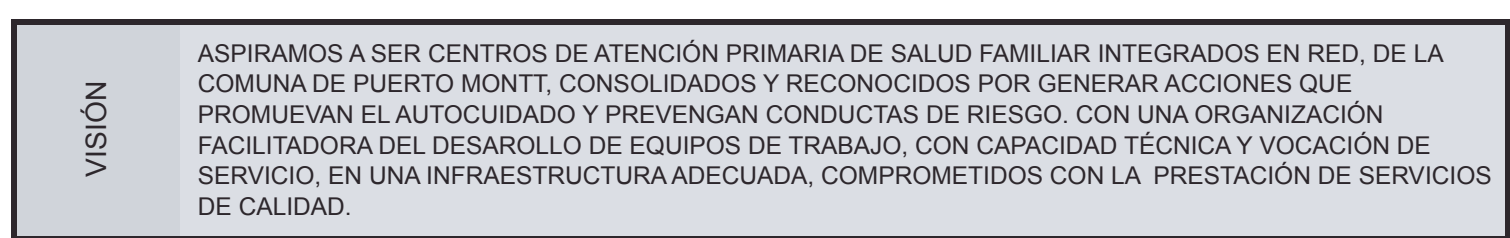

\section{Volver Atrás}




\section{FIGURA 5. Mapa estratégico}

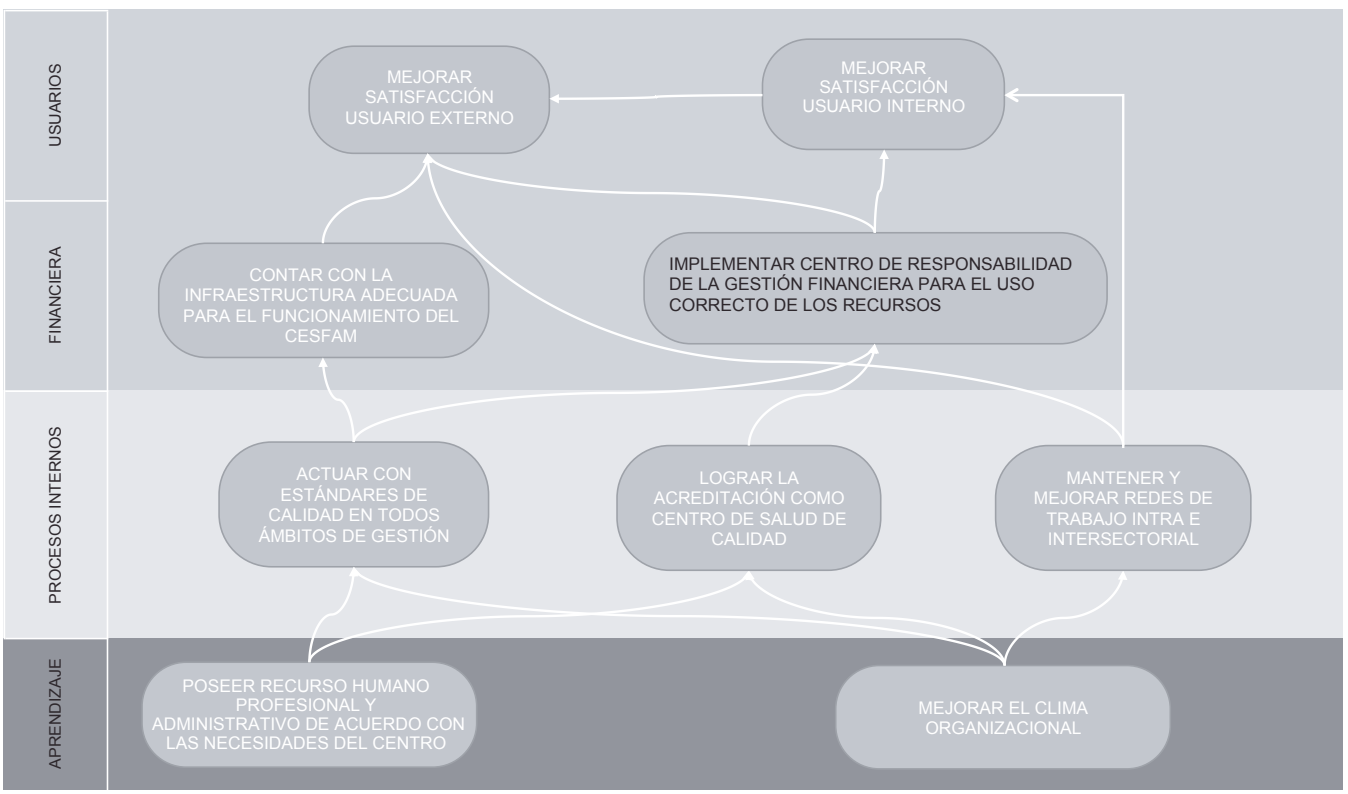

Fuente: elaboración propia

FIGURA 6. Perspectiva usuario

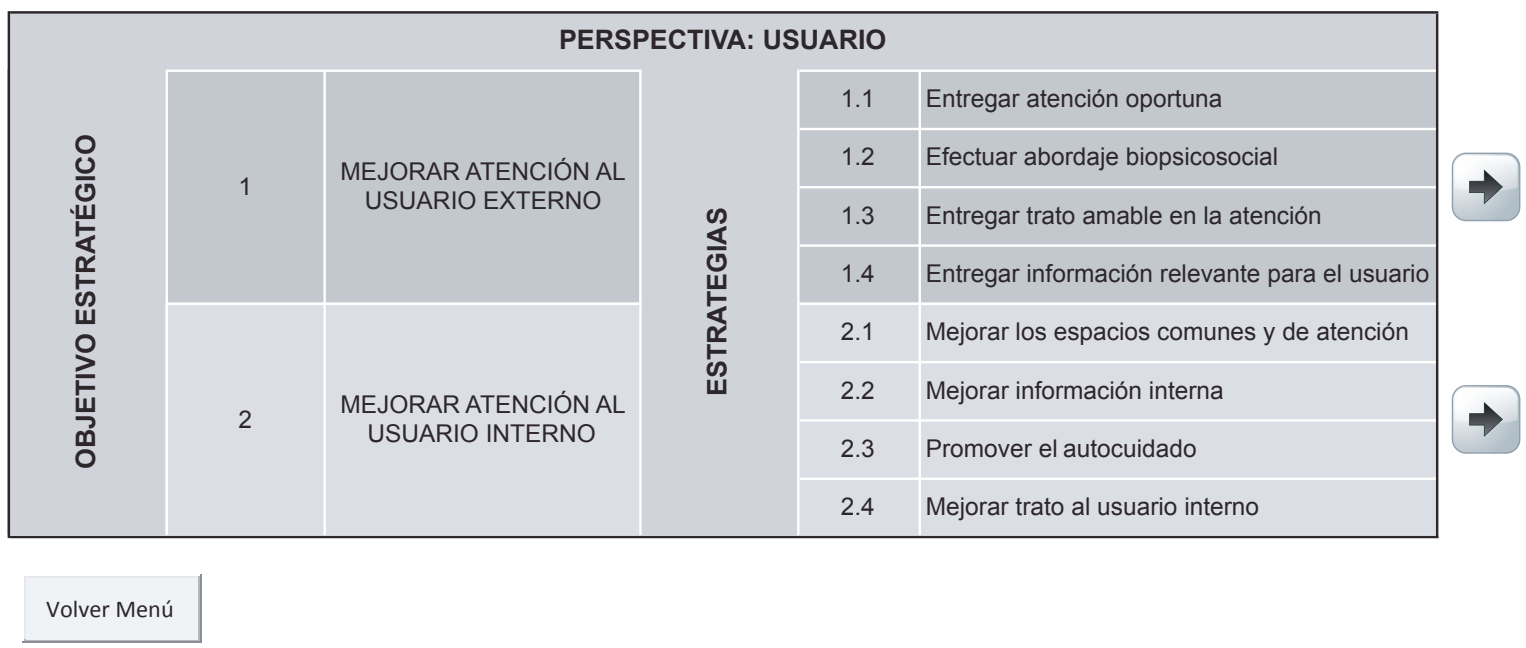

Fuente: elaboración propia 
FIGURA 7. Perspectiva financiera

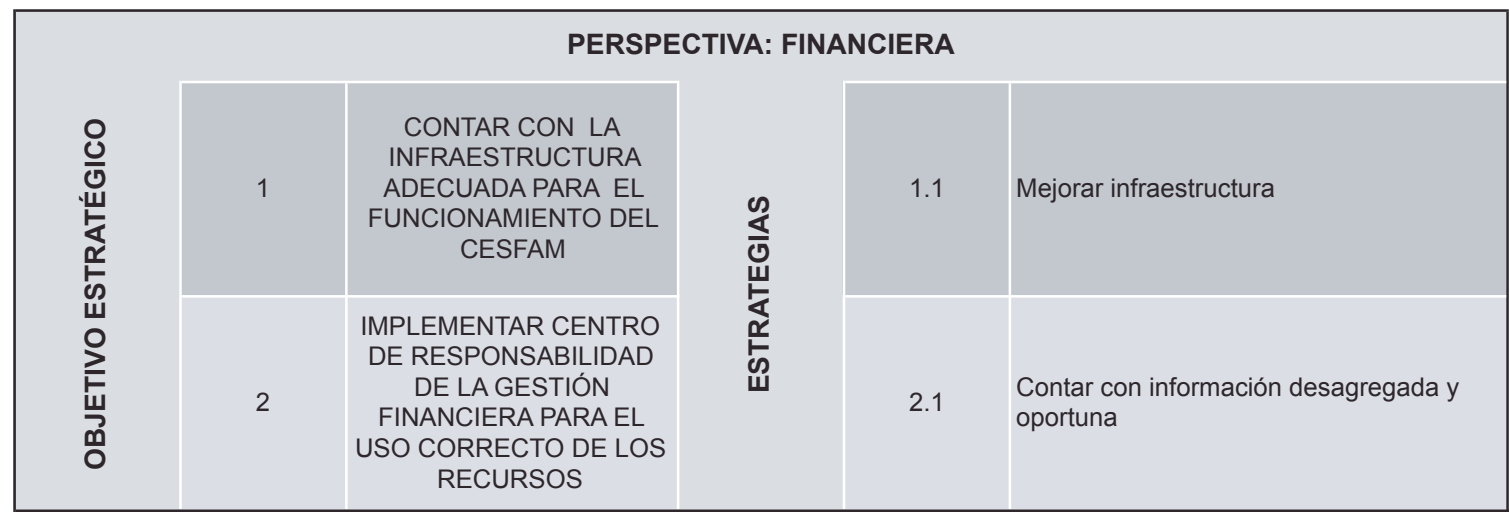

Fuente: elaboración propia

FIGURA 8. Perspectiva procesos internos

\begin{tabular}{|c|c|c|c|c|c|}
\hline \multicolumn{6}{|c|}{ PERSPECTIVA: PROCESOS INTERNOS } \\
\hline \multirow{10}{*}{ 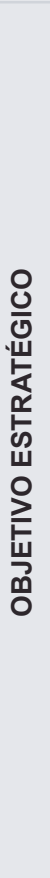 } & \multirow{7}{*}{1} & \multirow{7}{*}{$\begin{array}{c}\text { ACTUAR CON } \\
\text { ESTÁNDARES DE } \\
\text { CALIDAD EN TODOS LOS } \\
\text { ÁMBITOS DE GESTIÓN }\end{array}$} & \multirow{10}{*}{ 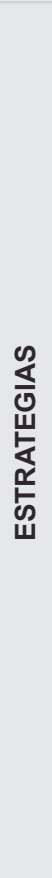 } & 1.1 & $\begin{array}{l}\text { Consolidar implementación de los sistemas de } \\
\text { registro informático y físico }\end{array}$ \\
\hline & & & & 1.2 & Levantar protocolos de atención y procesos \\
\hline & & & & 1.3 & Impulsar mejora continua \\
\hline & & & & 1.4 & Mejorar equipamiento del centro \\
\hline & & & & 1.5 & $\begin{array}{l}\text { Desarrollar investigación pertinente al quehacer } \\
\text { del Cesfam Angelmó }\end{array}$ \\
\hline & & & & 1.6 & $\begin{array}{l}\text { Generar las condiciones para el desarrollo de la } \\
\text { docencia asociada al quehacer del centro }\end{array}$ \\
\hline & & & & 1.7 & Captar convenios y proyectos \\
\hline & \multirow{2}{*}{2} & \multirow{2}{*}{$\begin{array}{c}\text { LOGRAR LA } \\
\text { ACREDITACIÓN COMO } \\
\text { CENTRO DE SALUD DE } \\
\text { CALIDAD }\end{array}$} & & 2.1 & Obtener acreditación del Cesfam Angelmó \\
\hline & & & & 2.2 & $\begin{array}{l}\text { Mantener la certificación de Cesfam Nivel } \\
\text { Superior }\end{array}$ \\
\hline & 3 & $\begin{array}{l}\text { MANTENER Y MEJORAR } \\
\text { REDES DE TRABAJO } \\
\text { INTRA E } \\
\text { INTERSECTORIAL }\end{array}$ & & 3.1 & Mantener redes de trabajo \\
\hline
\end{tabular}

\section{Volver Menú}

Fuente: elaboración propia 
FIGURA 9. Perspectiva aprendizaje

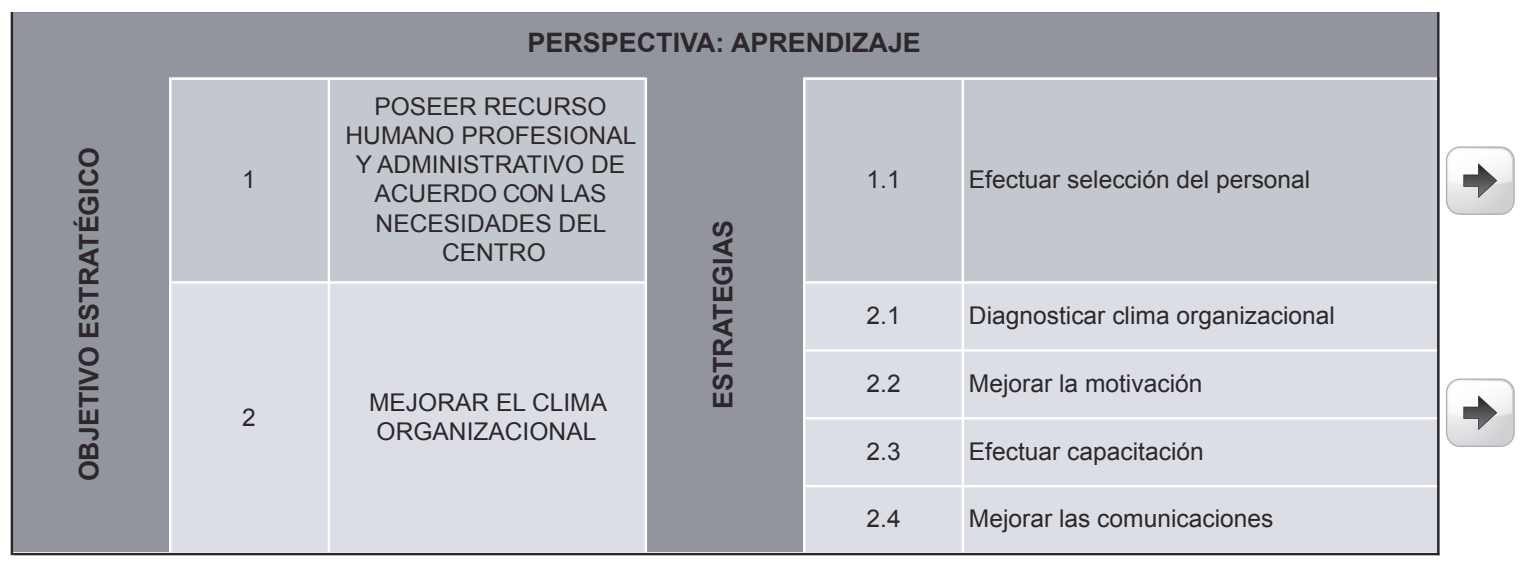

Volver Menú

Fuente: elaboración propia

FIGURA 10. Perspectiva usuario: objetivo, estrategia, acciones, indicadores, criterio de alerta, responsable y meta

\begin{tabular}{|c|c|c|c|c|c|c|c|c|c|}
\hline \multicolumn{7}{|c|}{ PERSPECTIVA: USUARIO } & & & \multirow{2}{*}{ Volver Atrás } \\
\hline \multicolumn{2}{|c|}{ OBJETIVO ESTRATÉGICO } & \multicolumn{5}{|c|}{ 1.- MEJORAR LA ATENCIÓN AL USUARIO EXTERNO } & & & \\
\hline \multirow[b]{2}{*}{1.1 .1} & RATEGIA & $\begin{array}{l}\text { ACCIONES PARA ALCANZAR } \\
\text { CUMPLIMIENTO DE LA META }\end{array}$ & INDICADORES & $\begin{array}{l}\text { CÁLCULOS DE } \\
\text { INDICADORES }\end{array}$ & $\begin{array}{l}\text { PERIOCIDAD DE } \\
\text { MEDICIÓN }\end{array}$ & \multicolumn{2}{|c|}{ CRITERIO DE ALERTA } & $\begin{array}{l}\text { RESPONSABLE } \\
\text { LOGRO DE LA META }\end{array}$ & META \\
\hline & \multirow[t]{2}{*}{$\begin{array}{l}\text { ENTREGAR } \\
\text { ATENCIÓN } \\
\text { OPORTUNA }\end{array}$} & $\begin{array}{l}\text { Gestionar la contratación de } 50 \% \\
\text { más de horas profesionales criticas } \\
\text { del área de salud. Considerando } \\
\text { contar con un médico de familia } \\
\text { por sector }\end{array}$ & $\begin{array}{c}\text { Porcentaje de nuevas } \\
\text { horas contratadas: } \\
\text { (Número de nuevas horas } \\
\text { contratadasas Número total } \\
\text { de horas actuales } \\
\text { (base))* } 100 \\
\text { Número de reuniones } \\
\text { Número de profesionales } \\
\text { criticos }\end{array}$ & \begin{tabular}{|c|}
$\begin{array}{c}N^{\circ} \text { de nuevas horas } \\
\text { contratadas }\end{array}$ \\
$\begin{array}{c}N^{\circ} \text { total de horas } \\
\text { actuales }\end{array}$ \\
\\
Porcentaje \\
\end{tabular} & Trimestral & \begin{tabular}{|c|}
$\begin{array}{c}\% \text { horas médicas } \\
\text { contratadas }\end{array}$ \\
$0 \%$ \\
inferior $50 \%$ \\
\end{tabular} & $\begin{array}{l}\text { Tipo de } \\
\text { alerta }\end{array}$ & $\begin{array}{l}\text { Director del } \\
\text { Cesfam/ } \\
\text { Direccín de Salud } \\
\text { Municipal (Disam) }\end{array}$ & $\begin{array}{l}\text { Al } 31 \text { de diciembre } \\
\text { de } 2014 \text {, se ha } \\
\text { gestionado la } \\
\text { contratación de } \\
50 \% \text { más de horas } \\
\text { profesionales } \\
\text { críticas del área de } \\
\text { salud y se cuenta } \\
\text { con médico de } \\
\text { familia por sector }\end{array}$ \\
\hline 1.1.2 & & $\begin{array}{l}\text { Disponer de } \\
\text { agendas de dación de horas } \\
\text { abiertas con } 6 \text { meses de } \\
\text { anticipación }\end{array}$ & $\begin{array}{l}\text { Número de horas } \\
\text { disponibles para dación } \\
\text { SI/ NO }\end{array}$ & & Mensual & $\begin{array}{c}\text { No es posible } \\
\text { disponer de } \\
\text { agendas }\end{array}$ & & Jefe de SOME & $\begin{array}{l}\text { A partir de } 2015 \text { se } \\
\text { dispone de } \\
\text { agendas de dación } \\
\text { de horas abiertas } \\
\text { con } 6 \text { meses de } \\
\text { anticipación }\end{array}$ \\
\hline
\end{tabular}

Volver Atrás

Fuente: elaboración propia 
FIGURA 11. Perspectiva financiera: objetivo, estrategia, acciones, indicadores, criterio de alerta, responsable y meta

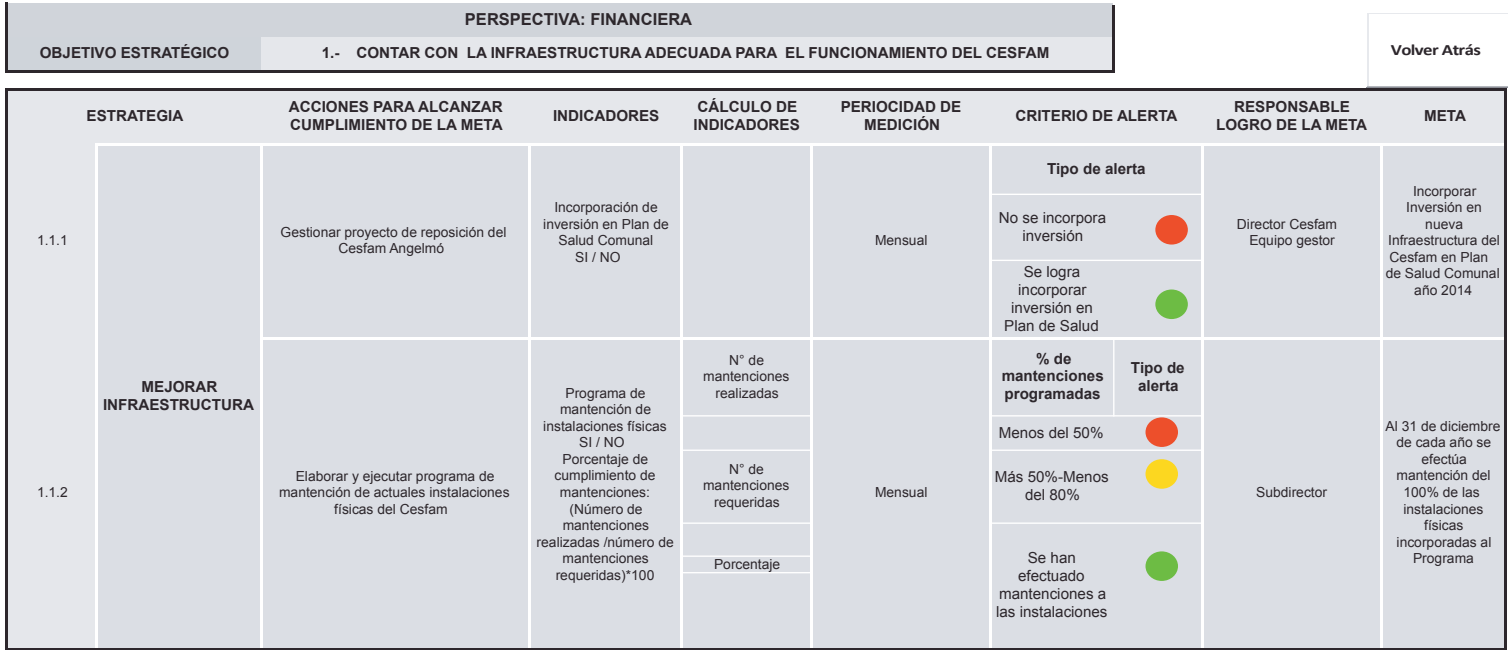

FIGURA 12. Perspectiva procesos internos: objetivo, estrategia, acciones, indicadores, criterio de alerta, responsable y meta

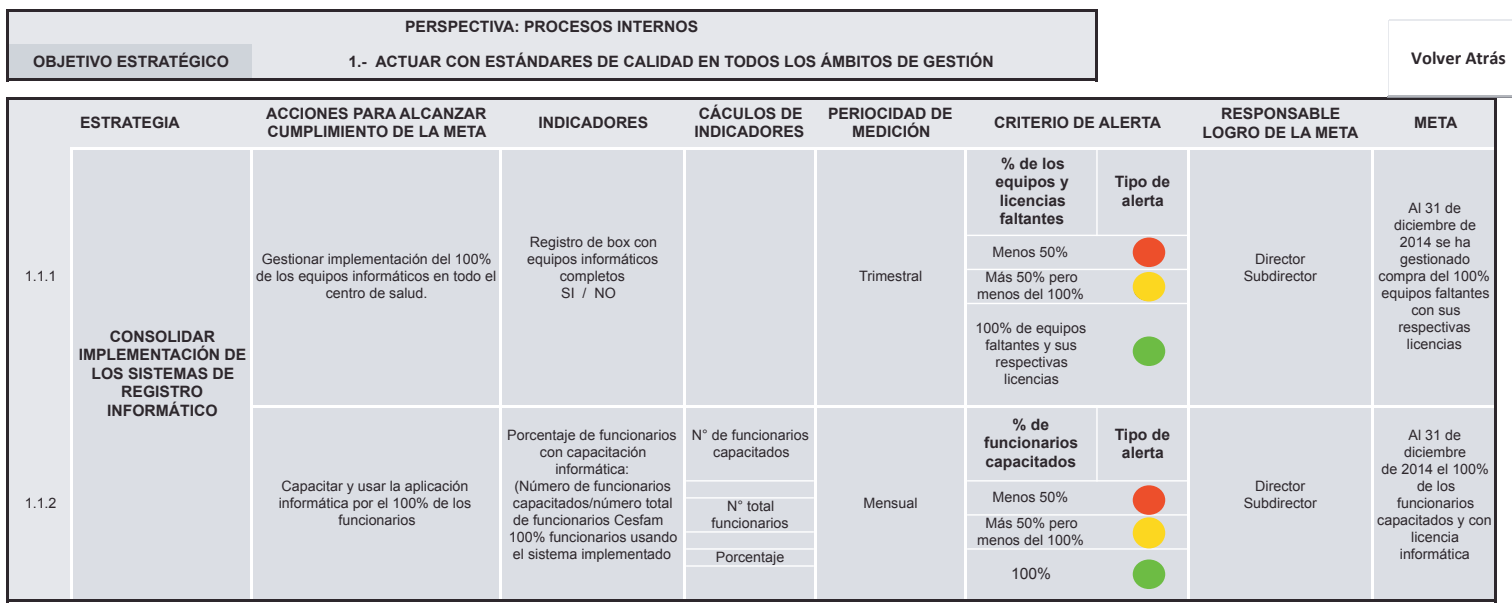


FIGURA 13. Perspectiva aprendizaje: objetivo, estrategia, acciones, indicadores, criterio de alerta, responsable y meta

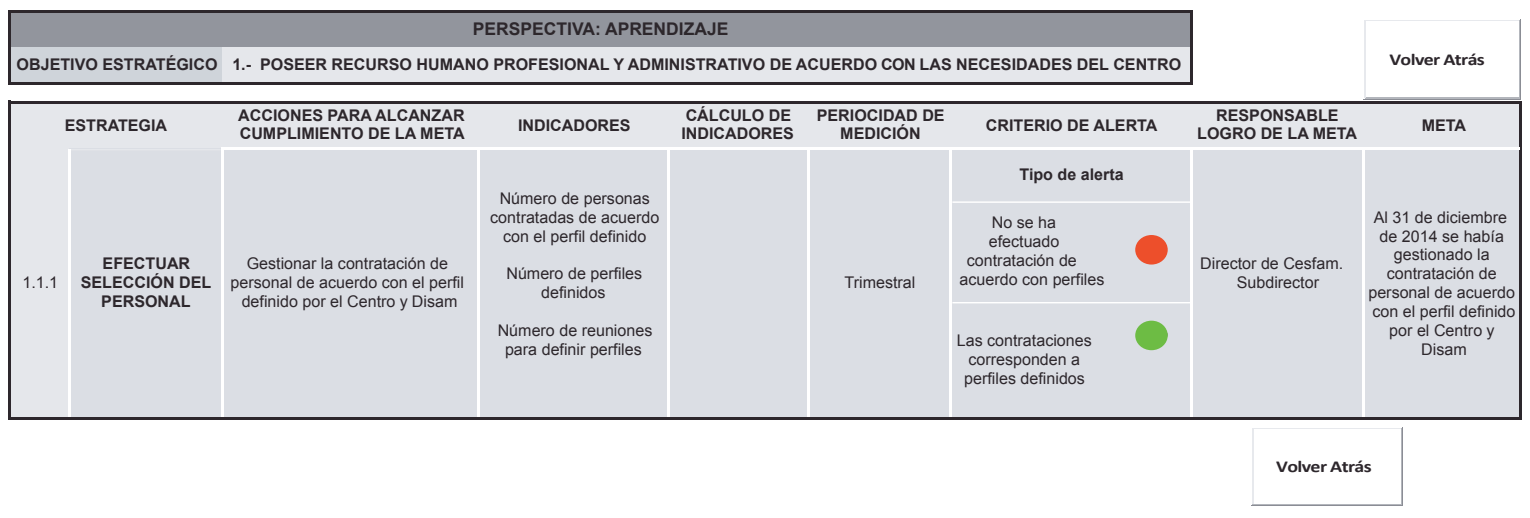

Fuente: elaboración propia

\section{Conclusión}

Se ha expuesto la aplicación del cuadro de mando integral en una organización de salud. Su diseño y puesta en marcha corresponde a cada Cesfam. En particular le corresponde a su director(a) y a su equipo gestor monitorear la ejecución de las acciones planificadas; no obstante, cada Cesfam debe incorporar dentro de su estructura organizacional una persona o unidad con la función de recolección, actualización periódica, sistematización y análisis de la información emanada por cada uno de los responsables del logro de las metas. El compromiso de los equipos gestores con el modelo brindará beneficios en las cuatro perspectivas.

Utilizar el BSC como metodología para el diseño de planificación estratégica permite orientación estratégica. El Balanced Scorecard hace posible una efectiva planificación, entender y comunicar la estrategia definida y gestionar mejor, con una visión global y de largo plazo. El proceso de evaluación y el instrumento constituyen un vehículo que direcciona las iniciativas estableciendo sinergias y una relación de causalidad entre las distintas perspectivas. Fue posible identificar las áreas de intervención y los procesos de mejora que se deben realizar de manera coherente, como también transmitir y comprometer a todos los estamentos.

\section{REFERENCIAS}

Armijo, M. (2009). Manual de Planificación Estratégica e Indicadores de Desempeño en el Sector Público (versión preliminar). Ilpes/Cepal. Recuperado de http://www.cepal.org/ilpes/noticias/paginas/3/38453/manual_planificacion_estrategica. pdf

Benavente, F. (2012). Balanced Scorecard: Una herramienta eficaz para la métrica de la estrategia en un Hospital Autogestionado. Recuperado de http:// web.minsal.cl/portal/url/item/b6456c5e21594a2 1e0400101650152a0.pdf

Hax, A. \& Majluf, N. (1996).Gestión de empresa con una visión estratégica. Santiago: Editorial Dolmen.

Kaplan, R. S. \& Norton, D. (1996). Cuadro de mando integral. Barcelona: Gestión 2000.

Leiva, H. \& Flamm, M. (2007). Cuadro de mando integral (BSC) del Servicio de Pediatría del Hospital Regional Rancagua. Revista Chilena de Salud Pública, 11(3), 142-149.

Ministerio de Salud. (2012). Orientaciones para la Implementación del Modelo de Atención Integral de Salud Familiar y Comunitaria dirigido a equipos de salud. Recuperado de http://web.minsal.cl/portal/ url/item/e7b24eef3e5cb5d1e0400101650128e9. pdf

Ministerio de Salud, Subsecretaría de Redes Asistenciales. (2008). En el camino a Centro de Salud Familiar. Recuperado de https://sochimef.files. wordpress.com/2014/12/minsal-2008-en-el-camino-a-centro-de-salud-familiar.pdf (2007).

Salinas M., López-Garrigos M., Gutiérrez M., Lugo J. \& Uris J. (2012). Las perspectivas financiera y de aprendizaje y crecimiento del cuadro de mando integral en las instituciones públicas: Aplicación en el laboratorio clínico. Gac Sanit., 26(1), 97-98. Recuperado de http://scielo.isciii.es/pdf/gs/ v26n1/carta1.pdf

Superintendencia de Salud. (2009). Manual del Estándar General de Acreditación para Prestadores Institucionales de Atención Abierta. Recuperado de http://www.supersalud.gob.cl/568/articles-4530_Manual_AA_pdf.pdf 